\title{
A calculated response: control of inflammation by the innate immune system
}

\author{
Gregory M. Barton
}

Division of Immunology, Department of Molecular and Cell Biology, University of California, Berkeley, Berkeley, California, USA.

\begin{abstract}
Inflammation is a rapid yet coordinated response that can lead to the destruction of microbes and host tissue. Triggers capable of inducing an inflammatory response include tissue damage and infection by pathogenic and nonpathogenic microbes. Each of these triggers represents a qualitatively distinct stress to the host immune system, yet our understanding of whether they are interpreted as such remains poor. Accumulating evidence suggests that recognition of these distinct stimuli converges on many of the same receptors of the innate immune system. Here I provide an overview of these innate receptors and suggest that the innate immune system can interpret the context of an inflammatory trigger and direct inflammation accordingly.
\end{abstract}

\section{Introduction}

Inflammation is a coordinated process induced by microbial infection or tissue injury $(1,2)$. The process involves an enormous expenditure of metabolic energy, damage and destruction of host tissues, and even the risk of sepsis, multiple organ failure, and death. The main function of inflammation is to resolve the infection or repair the damage and return to a state of homeostasis. Intrinsic to the efficacy of such a system is the ability to mount a rapid response appropriate to the particular type of inflammatory trigger while limiting the damaging aspects of inflammation as much as possible. Thus the ideal inflammatory response is rapid and destructive (when necessary), yet specific and self limiting. The importance of this balance is demonstrated by the observations that in certain chronic infections or inflammatory disorders the inflammatory response causes more damage to the host than the microbe $(2,3)$.

Defining the signals that orchestrate coordinated inflammatory responses is a clinically relevant problem with implications for our overall understanding of how the immune system senses stress in the form of infection and tissue damage. At the most basic level, the immune system can be viewed as two interconnected branches. Innate immunity is the more ancient of these branches, existing, in some form, in all metazoan organisms (4). Cells of the innate immune system use invariant receptors to detect and signal the occurrence of microbial infection $(4,5)$. These signals serve two principal functions: initiation of an inflammatory cascade that helps contain the infection and activation of the adaptive immune response, the second branch of the immune system. The adaptive immune response consists of expansion of the small number of microbial-specific lymphocytes, a process that is highly effective and specific but that takes days to fully develop. Therefore, the hallmarks of inflammation that we associate with microbial infection (i.e., redness, heat, swelling, and pain) are initiated by innate immune recognition. Cells of the adaptive immune response can

Nonstandard abbreviations used: HMGB1, high-mobility group box 1; IL-1R, receptor for IL-1; LRR, leucine-rich repeat; Naip, NLR family apoptosis inhibitory protein; Nalp, NACHT-, LRR-, and pyrin domain-containing protein; NLR, Nod-like receptor; Nod, nucleotide-binding oligomerization domain; PRR, pattern-recognition receptor; RIG-I, retinoic acid-inducible gene I; RLR, RIG-I-like receptor; SWI/SNF, switching-defective/sucrose non-fermenting; TIR, Toll/IL-1 receptor.

Conflict of interest: The author has declared that no conflict of interest exists. Citation for this article: J. Clin. Invest. 118:413-420 (2008). doi:10.1172/JCI34431. contribute to and exacerbate these effects, but the primary signals that start the response and ultimately resolve it are linked to innate immune recognition.

In this article, I describe the signals that are integrated into the decision to initiate an inflammatory response. In particular, I focus on whether the host distinguishes different inflammatory triggers and, if so, how this discrimination is mediated. Tissue damage (i.e., injury) and microbial infection are the primary inducers of inflammation $(1,2)$. The role for innate receptors in detecting microbial infection is now well appreciated (4), although whether the host can discriminate between pathogenic and nonpathogenic microbes is less clear. Recent work suggests that certain products of tissue damage can also be detected by innate receptors $(6,7)$. This convergence of proinflammatory triggers on innate immune receptors raises the question of whether sterile injury and microbial infection are viewed equivalently by the innate immune system. The benefits of such a design are difficult to envision. Although it might be emotionally appealing to group inflammatory stimuli together with amorphous labels such as "danger signals" (8), the reality is that infection and tissue damage present qualitatively different challenges to the host. At the most basic level, the former involves the presence of a foreign organism, whereas the latter does not. Accordingly, the appropriate response to infection might require additional destruction of self tissue in order to contain the microbe $(1,9)$. This response seems to escalate if a pathogen has acquired virulence mechanisms that enable it to manipulate the host response $(10,11)$. In contrast, the response to tissue damage might focus more on repair. Based on the recent advances in our understanding of innate immune signaling discussed here, I propose that distinct inflammatory outcomes might depend on the type of ligand recognized and the context in which recognition takes place.

\section{Induction of inflammation through pattern recognition}

The main trigger of inflammation is the recognition of microbes by receptors of the innate immune system (4). This molecular recognition event is conceptually quite distinct from the recognition of microbial components by antigen receptors of $\mathrm{T}$ and $\mathrm{B}$ lymphocytes. Lymphocytes collectively express billions of unique, randomly generated receptors and therefore have the capacity to recognize any target (12). In contrast, the specificities of innate receptors are "hardwired," meaning their microbial targets are selected over evolutionary time and, by definition, are limited in 


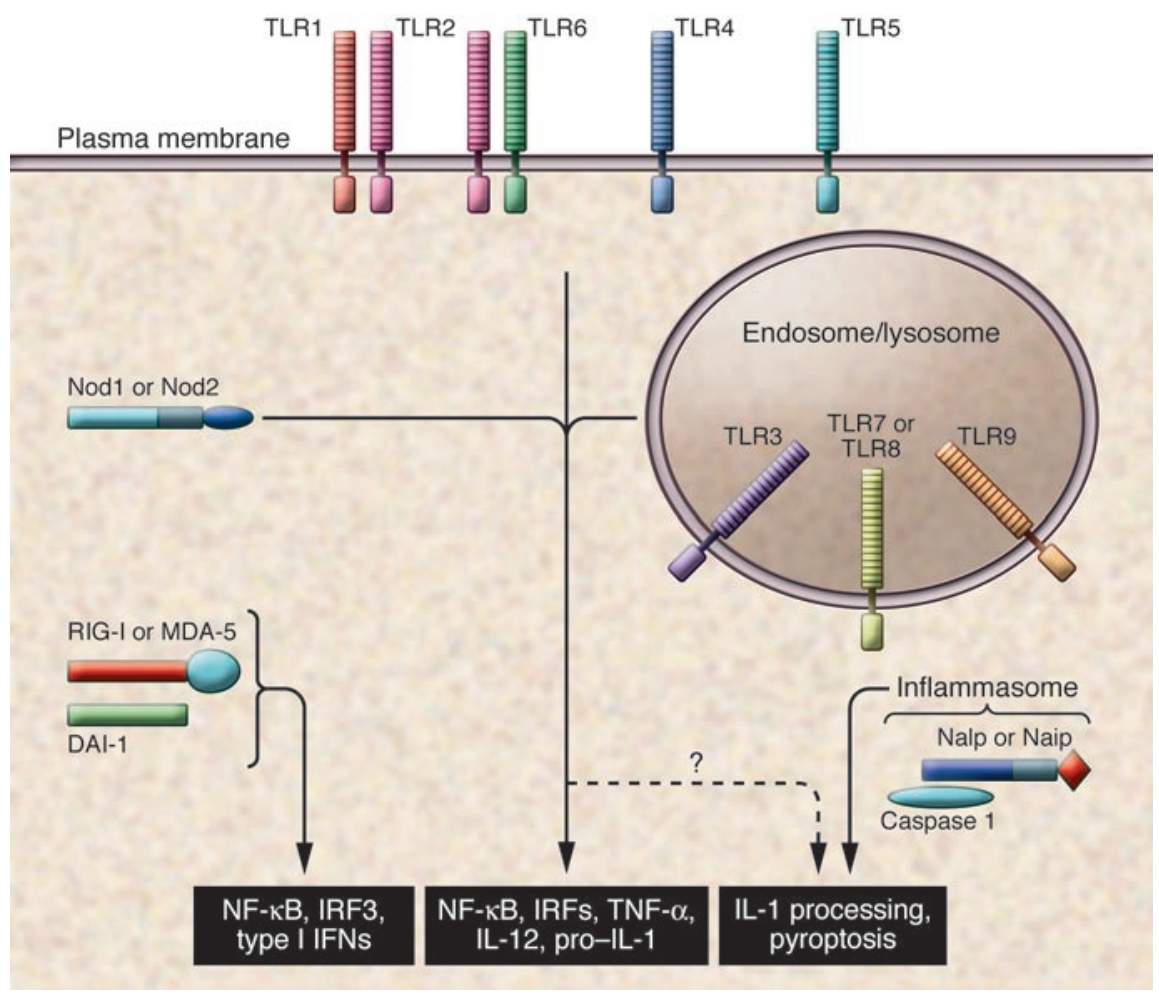

\section{Figure 1}

Pattern recognition by the innate immune system. A schematic representation of the PRR families within the innate immune system. TLRs are transmembrane receptors, whereas Nods, Naips, Nalps, as well as the nucleic acid sensors RIG-I, MDA-5, and DAI- 1 are all present in the cytosol. Most of these PRR families activate common transcription factors and induce the expression of proinflammatory genes. Members of the Nalp and Naip families control activation of the inflammasome, a multiprotein complex responsible for the processing and secretion of IL-1. Whether other PRR families can directly induce inflammasome activation remains controversial. Refer to the text for a discussion of the ligands recognized by each PRR family. scope (13). This restriction has shaped the specificities of innate receptors to ensure that the many different classes of potential pathogens can be recognized (14). By targeting highly conserved features of microbes, innate receptors are able to detect the wide range of microbial diversity $(15,16)$. This strategy has been termed "pattern recognition" and the innate receptors employing it "pattern-recognition receptors" (PRRs). Examples of microbial patterns are LPS and peptidoglycan from the cell walls of gram-negative and gram-positive bacteria, respectively.

PRRs are involved in multiple aspects of mammalian immune systems $(14,17)$. They can function as phagocytic transmembrane receptors (e.g., the mannose receptor and dectin-1) or soluble proteins involved in opsonization and complement activation (e.g., mannose-binding lectin) (18). Most relevant to the initiation of inflammation, though, are PRRs that link microbial recognition to proinflammatory signals (Figure 1). The best characterized of these gatekeepers of inflammation are the TLRs, an ancient family of transmembrane proteins (15). All TLRs contain an extracellular domain with a series of leucine-rich repeats (LRRs) and an intracellular domain with a conserved signaling module called a Toll/IL-1 receptor (TIR) domain. TLRs are expressed by phagocytic cells, such as macrophages, neutrophils, and DCs, and they link recognition of conserved components of bacteria, viruses, fungi, and protozoa to activation of these cells $(15,16)$. The growing list of TLR ligands includes LPS (TLR4); peptidoglycan, lipoteichoic acid, and zyomosan (TLR2); double-stranded RNA (TLR3); flagellin (TLR5); single-stranded RNA (TLR7 and TLR8); and nonmethylated CPG motifs in DNA (TLR9). Activation initiates a conserved signaling cascade that culminates in the activation of NF- $\mathrm{\kappa B}$ and IFN-regulatory factor (IRF) transcription factors (19). These factors drive the expression of proinflammatory genes (such as TNF- $\alpha$ and IL-1) and initiate the production of signals that initiate adaptive immunity.
In addition to the membrane-spanning TLRs, a large family of cytosolic PRRs participates in the detection of pathogens that are able to enter host cells $(20,21)$. Understanding the role that this large family plays in host defense is an area of intensive research with many unanswered questions. These receptors are more varied in their structure and function than the TLRs, yet they are collectively referred to as nucleotide-binding oligomerization domain-like (Nod-like) receptors (NLRs), based on their structural similarity to the Nod 1 and Nod 2 proteins (4). NLRs share a similar domain structure, including an LRR domain, which mediates ligand recognition; a nucleotide-binding domain, which mediates dimerization; and a signaling module. The signaling modules dictate the functional outcome of NLR activation. For Nod1 and Nod2, this function seems somewhat similar to that of TLRs in that these receptors activate NF- $\mathrm{\kappa B}$ and induce the expression of proinflammatory cytokines (22). In fact, in certain circumstances, activation of either Nod1 or Nod2 can synergize with TLR signaling, leading to enhanced cytokine production (23).

The function of other NLR family members is distinct from the functions of Nod1 and Nod2. A subset of the NLR family, which includes the NLR family apoptosis inhibitory proteins (Naips) and NACHT-, LRR-, and pyrin domain-containing proteins (Nalps), controls activation of the inflammasome, a multiprotein complex involved in activating caspase-1, a protease that processes pro-IL-1 into a mature active form that is then secreted (11). Naips and Nalps recognize bacteria (and possibly other classes of microbes) by targeting patterns similar to those recognized by TLRs (11). Ligands implicated in the activation of these receptors are quite diverse, including bacterial RNA, uric acid crystals, bacterial toxins, and flagellin (11). In contrast to TLRs, however, these ligands must reach the cytosol, either because the bacteria has crossed the cellular membrane or because the bacteria is able to inject proteins across this membrane. 


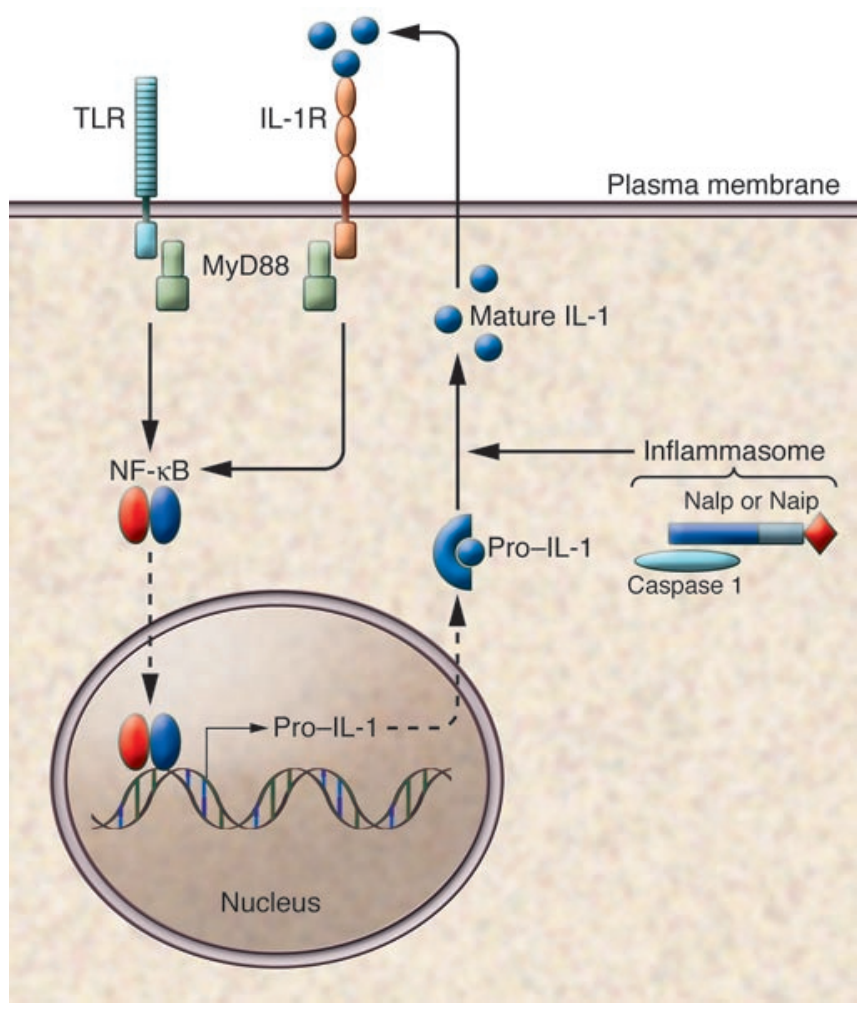

Activation of the inflammasome leads to the secretion of IL-1, a potent proinflammatory cytokine that mediates many of the early signals that establish an inflammatory state $(4,11)$. In some cell types, activation of the inflammasome also induces rapid cell death. Sometimes referred to as pyroptosis, this form of cell death lacks many of the hallmarks associated with apoptosis $(10,24)$. Consequently, death by pyroptosis is thought to be more inflammatory than death by apoptosis (10). It has been suggested that rapid induction of cell death helps prevent bacterial replication $(25,26)$.

The function of TLR and NLR pathways are interconnected at multiple points (Figure 2). As mentioned above, Nod1 and Nod2 can synergize with TLRs to enhance cytokine production (23). In addition, TLR activation regulates the activity of the inflammasome. First, TLR signaling is required to induce expression of pro-IL-1 as well as components of the inflammasome itself (11). Without this TLR "priming," activation of the inflammasome does not lead to significant IL-1 secretion, regardless of whether caspase- 1 is activated (11). A second link comes from the fact that the receptor for IL-1 (IL-1R) signals via a cytosolic TIR domain (similar to the TIR domain of TLRs) and activates many of the same downstream signaling pathways as TLRs (5). In this sense, IL-1 might be considered the equivalent of an endogenous TLR ligand whose induction is controlled by NLR activation. In fact, many of the proinflammatory features attributed to TLR activation might be partially dependent on IL-1R signaling, especially considering that MyD88-deficient mice cannot signal through either type of receptor (27). In any case, it seems that the TLR and NLR pathways reinforce each other at multiple points.

A final group of cytosolic PRRs involved in sensing nucleic acid has been more recently discovered. The first of these receptors to be identified was retinoic acid-inducible gene I (RIG-I) (28), and these receptors are collectively referred to as RIG-I-like receptors (RLRs)

\section{Figure 2}

Cross-talk between TLR and NLR pathways. The TLR and NLR families sense microbial ligands in different compartments within the cell. NLRs are activated by the presence of cytosolic microbes or the action of microbial virulence mechanisms. These signals feed back on the TLR transcriptional response by activating IL-1R, which activates many of the same signaling pathways as TLRs.

$(29,30)$. RIG-I and melanoma differentiation-associated protein-5 (MDA-5) are PRRs with RNA helicase domains that detect foreign RNA in the cytosol. Foreign DNA is also sensed by the innate immune system (31-33). The molecular details of this pathway are less well characterized, although a protein called DNA-dependent activator of IFN-regulatory factors (DAI) was recently implicated in the recognition of foreign DNA in the cytosol (34). Both the RNA and DNA sensing pathways induce type I IFNs and contribute to the induction of an antiviral state $(29,30)$. It also seems that bacterial nucleic acid can activate these pathways, although how this activation occurs and its relevance for the immune response to bacteria is less clear $(33,35,36)$. There is not yet any evidence that these pathways contribute to inflammasome activation, nor that there is cross-talk between TLRs and RLRs.

\section{The core innate immune response: inflammation to infection}

Inflammation is a general term used to describe the cascade of events that occurs in response to infection or tissue damage (1). The study of this process predates the modern era of molecular biology, and consequently many of the hallmarks of inflammation involve qualitative measures of cellular responses. However, the last several decades have seen an enormous increase in our understanding of the molecular pathways controlling inflammation and the early response to microbial infection $(4,5)$. In particular, TLRs and other PRRs play a crucial role in the induction of early signals that establish the inflammatory setting $(2,4)$. In this section I attempt to integrate our growing understanding of innate immune pathways into the complex framework of an inflammatory response. For the discussion to remain conceptually useful, I have chosen not to discuss the details of inflammation in great depth. This topic has been covered in detail elsewhere (2). Instead, I focus on what I consider the key regulatory steps in inflammation and how information conveyed by innate immune receptors can contribute at these steps.

The inflammatory response to infection has traditionally been classified as 4 distinct phases: recognition of infection, recruitment of cells to the site of infection, elimination of the microbe, and resolution of inflammation and return to homeostasis (1). Layered on this local inflammatory response is the induction of adaptive immunity, which occurs as DCs and antigen drain from the site of inflammation to regional lymph nodes (37). The innate and adaptive responses are intricately linked, and many of the signals discussed herein as they relate to inflammation are also important for efficient lymphocyte activation. Space considerations preclude a detailed discussion of these aspects of downstream innate signals.

Recognition of infection. Multiple cell types that are resident in tissues prior to infection express PRRs that are capable of recognizing microbial organisms. Most influential in the early sensing of infection are tissue-resident macrophages and DCs (37). Mast cells have also been implicated in the early phases of inflammation 
through their release of cytokines and preformed mediators such as histamine, prostaglandins, and leukotrienes (38). Macrophages and DCs engulf microbes, which they detect via PRRs that are expressed on their cell surface, within endosomal/lysosomal compartments, and in the cytosol $(4,37)$. The net result of pathogen recognition is activation of resident phagocytes and mast cells and the release of proinflammatory cytokines and preformed mediators. The pleiotropic effects of these molecules begin a cascade of events that will profoundly change the local environment of the surrounding tissue and vasculature $(1,2)$.

Recruitment of inflammatory cells to the site of infection and elimination of the microbe. Cytokines and other proinflammatory mediators induce changes in local blood vessel endothelial cells that promote the conversion of the infected tissue to an inflamed state. In particular, TNF- $\alpha$, IL-1, and lipid mediators cause a series of morphological and molecular changes that collectively lead to increased migration of leukocytes and flow of plasma to the infected site. In most cases, neutrophils arrive within hours followed by a later influx of monocytes (9). Neutrophils enter the inflamed site armed with a battery of effector mechanisms that can wreak havoc on microbial organisms as well as host tissues (9). Not only do they possess granules loaded with proteases capable of degrading phagocytosed material, but they also produce ROS and reactive nitrogen species (RNS) that denature proteins, disrupt lipids, and damage DNA (39). After entering the site of inflammation, neutrophils phagocytose any available microbes and direct the contents of their granules toward these phagosomes (9). If neutrophils detect TNF- $\alpha$ but do not directly encounter any microbial particles after entering the tissues, they release their granules into the extracellular space in an effort to create an inhospitable environment for nearby pathogens (9). The contents of these granules are not uniquely toxic for microbial organisms and generate significant damage to host tissues and cells. For example, elastase, cathepsin $\mathrm{G}$, and proteinase 3 are broad specificity serine proteases that can break down components of the extracellular matrix and destroy host cells leading to liquefaction of tissue. This toxicity is neither random nor accidental; it serves to contain the infection in the critical early phase before the full immune response has mounted. Mice lacking these proteases are highly susceptible to bacterial infections due to their inability to contain bacterial spread in the early phases of infection (40-42). All options for neutrophils, however, result in the same eventual fate: their death by apoptosis and clearance by macrophages. As mentioned earlier, these macrophages enter the site of inflammation after neutrophils, called by many of the same signals. In addition to the clearance of apoptotic neutrophils, macrophages also contribute to the killing of microbial organisms. They engulf and degrade microbes using proteases, antimicrobial peptides, ROS, and RNS.

Resolution of inflammation. If the inflammatory response is able to contain the microbial infection, then the overall response is shifted toward antiinflammatory signals and resolution of inflammation (43). Identification of the signals mediating this shift to resolution and repair is an area of active research, although several families of lipid-derived mediators play a clear role. In particular, lipoxins, protectins, and resolvins send antiinflammatory signals that actively promote resolution and tissue repair (44). The best characterized of these compounds are lipoxins, arachidonic acid (AA) derivatives generated by lipoxygenases (45). Lipoxins stop the influx of neutrophils, promote the uptake of apoptotic neutrophils by macrophages, and recruit additional monocytes to help clear away dead cells and tissue debris. How this switch to the antiinflammatory, pro-resolving phase of the inflammatory response is regulated remains unclear. One mechanism seems to be induction of lipoxins and protectins by macrophages that have ingested apoptotic neutrophils (45). In addition to these resolution mediators, macrophages, neutrophils, and epithelial cells produce secretory leukocyte protease inhibitor (SLPI) (46). SLPI inactivates the proteases released from neutrophil granules and pushes the inflammatory response toward resolution (47).

\section{Sterile inflammation}

Cells that die during tissue damage can trigger an inflammatory response that shares many features with inflammation induced by infection (1). For the sake of clarity, it is important to distinguish sterile injury from injury with associated infection, as these situations are conceptually distinct. The latter should be considered equivalent to microbial infection. In fact, most injuries are associated with concomitant infection, as they involve the disruption of a physical barrier, such as cuts or burns that enable microbes to bypass the skin. In certain cases, however, injury and cell death can occur in a sterile setting (48-51). The most common causes are trauma, ischemia, and ischemia-reperfusion. Even though these inflammatory triggers occur in the absence of infection, they induce many of the events described above, including DC activation and neutrophil and monocyte infiltration $(27,52)$. In severe instances, the inflammation associated with these injuries can escalate into circulatory shock and multiple organ failure (48). Precisely how tissue injury is detected in the absence of infection and what molecular events lead to inflammation are surprisingly poorly defined. A growing body of literature, however, implicates TLRs and other innate receptors in the detection of sterile injury $(49,50,53)$.

A number of endogenous molecules that are released when cells die by necrosis have been proposed to be indicative of sterile cell injury. These endogenous ligands include heat shock proteins, hyaluronan, $\beta$-defensin, uric acid crystals, and numerous other biomolecules $(6,49-52,54-62)$. Although TLR family members have been implicated in the recognition of these ligands, such studies are always plagued by the specter of microbial contamination as the basis for the stimulation (63). Still, it is becoming increasingly difficult to dismiss the stimulatory capacity of all endogenous ligands as the result of microbial contamination. Perhaps the strongest evidence that TLRs play a role in the detection of tissue injury comes from studies of TLR-deficient mice using various models of tissue injury. Using a sterile lung injury model, Noble and colleagues have demonstrated a role for TLR2 and TLR4 in mediating inflammation and tissue repair (49). In this system, TLR 2 and TLR4 seem to recognize damaged extracellular matrix by binding breakdown products of hyaluronan, a large nonsulfated glycosaminoglycan that can be cleaved during tissue damage. Additional support for TLR-mediated detection of injury comes from models of hemorrhagic shock and bilateral fractures $(48,50)$. TLR4-deficient mice mount substantially weaker inflammatory responses in these models of traumatic sterile injury. Precise characterization of the endogenous TLR ligands in these models is still lacking, but it is difficult to argue with the strong in vivo phenotypes of TLR-deficient mice in these studies.

Apart from these models of sterile tissue injury, deciphering the role of endogenous TLR ligands in inflammation is difficult. Although numerous in vitro studies have identified endogenous 
ligands that can stimulate TLRs, without clear in vivo evidence it is difficult to rule out microbial contamination. Nucleic acid ligands are the possible exception to this generalization. Host RNA and DNA are released from necrotic cells during tissue injury and infection-induced inflammation (64). These nucleic acids can serve as ligands for certain TLR family members, most notably TLR7, TLR8, and TLR9 (64), and certain endogenous proteins seem to play a role in delivering these ligands to TLRs in endosomes and lysosomes. In particular, the nuclear DNA-binding protein high-mobility group box 1 (HMGB1) has been implicated in TLR activation in certain inflammatory settings $(65,66)$. One model for HMGB1 function in inflammation stems from its ability to bind, stabilize, and deliver genomic DNA released from necrotic cells to $\operatorname{TLR} 9(65,66)$. This model would seem to make sense, as HMGB1 is released from necrotic cells and in some cases is secreted by activated macrophages. In mouse models of endotoxic shock as well as in human septic patients, elevated HMGB1 levels have been observed in the serum. Remarkably, neutralizing antibodies directed against HMGB1 can reduce the lethality associated with sepsis in these situations, indicating that HMGB1 functions as a late mediator of lethality (67). Perhaps HMGB1 serves to amplify the inflammatory reaction during sepsis by stimulating TLR9. One confusing fact that must be noted is that several groups have reported that HMGB1 can activate TLR4 and TLR2 directly $(59,61)$. Whether these reports represent yet another example of microbial contamination of an endogenous ligand, as opposed to another layer of complexity, remains to be seen. In any case, these data complicate the simple model in which the ability of HMGB1 to promote inflammation is due to delivery of self DNA to TLR9. Regardless of the precise mechanism, however, there is ample evidence to support the view that HMGB1 acts as an endogenous signal for tissue damage.

A final example of how endogenous molecules might be recognized by TLRs comes from a recent report describing how the antimicrobial peptide LL37 can deliver self nucleic acid to TLR9 (56). LL37 seems capable of condensing DNA as a way to facilitate DNA uptake into TLR9-containing endosomes (56). In psoriasis, LL37 can lead to TLR9-dependent production of type I IFNs, and this induction contributes to the immunopathology of the disease (56). However, whether LL37 can serve as a facilitator of TLR recognition of self DNA in the normal homeostasis of tissue injury and repair remains to be seen.

\section{Degrees of inflammation: tissue injury, microbes, and pathogens}

If induced or regulated improperly, the elements of inflammation outlined above have the capacity to cause catastrophic damage to the host. The destructive potential of this response is vital for survival during microbial infection but also causes substantial collateral damage. Thus a complex network of regulatory signals determines the appropriate degree of inflammation based on initial and ongoing cues. This coordination of inflammation has been described as a series of "stop and go" signals that determine when to escalate, dampen, or resolve the response (2). As discussed above, both infection and tissue injury trigger inflammatory responses but represent very distinct problems for the host. Nevertheless, the ligands that signal each event seem to converge on similar innate pathways (discussed above). This apparent lack of discrimination between such qualitatively different stresses to the host is confusing and suggests that our understanding of these processes is incomplete. Based on the complex feedback mecha- nisms regulating inflammation, it would seem that the capacity to regulate inflammation to these distinct stimuli should exist, yet there is little data to address this fundamental issue. Pathogenic microbes (i.e., microbes that have acquired virulence mechanisms) would seem to represent a third qualitatively distinct challenge, which necessitates an escalated response. A system in which inflammation escalates or dampens based on the nature of the trigger is conceptually appealing, yet our understanding of innate immune signaling cannot yet account for such specificity. However, recent advances in our understanding of specific gene induction downstream of innate receptors suggest that such a scenario may not be so farfetched (68-70).

Do tissue injury and microbial infection represent distinct stresses to the host? The extent to which the endogenous ligands produced during tissue damage recapitulate the inflammatory signal induced by microbes remains unclear. It is formally possible that the responses to tissue damage and infection are equivalent, perhaps due to the high likelihood of concomitant infection during tissue damage. As mentioned above, severe trauma can lead to hemorrhagic shock much like overwhelming infection can lead to sepsis. Nevertheless, these severe examples might misrepresent whether the overall purpose of each response is similar. Indeed, it is difficult to envision why the response to tissue damage would be identical to the response to infection. In the former, the goal is to repair, whereas the latter requires additional damage before healing can begin. Tissue repair requires tissue remodeling, breakdown of extracellular matrix, and proliferation of cells to reestablish homeostasis, and many of these aspects overlap with features of the resolving phase of inflammation in response to infection (1). Much of the collateral damage caused by activated neutrophils and macrophages during microbial infection might be unnecessary to heal a sterile wound.

The problem with the conceptual argument I make above is that, as discussed earlier in this Review, both tissue injury and microbial infection converge on the same set of receptors. If the responses to these triggers are distinct, then at a molecular level we must consider a model in which the same receptor induces distinct signal transduction and gene expression when activated by an endogenous ligand versus a microbial ligand (Figure 3 ). Such phenomena have been observed for TLR2 and TLR4. A study examining the transcriptional response to necrotic cells found that necrotic cells can induce a TLR2-dependent transcriptional response that is distinct from the response induced by microbial TLR2 ligands (71). Notably, necrotic cells induced the expression of genes involved in tissue repair, such as those encoding VEGF and MMP-3 (also known as stromelysin), as well as those encoding chemokines involved in neutrophil and macrophage recruitment (including $\mathrm{KC}$ and macrophage inflammatory protein 2 ). In contrast, microbial TLR2 ligands induced a distinct response, including the proinflammatory cytokine IL-12. TLR4 also seems capable of distinct signaling in response to different ligands. In response to vesicular stomatitis virus $G$ protein (VSV-G), TLR4 induced only a subset of the downstream signaling pathways activated by LPS (69). Thus an individual TLR is capable of differential signaling in response to different ligands, although the mechanisms responsible for these distinct responses remain undefined.

In light of these data, it seems possible that endogenous TLR ligands released during injury might induce a transcriptional response that is distinct from the response induced by microbial ligands. In the context of an infection, these endogenous ligands 
i

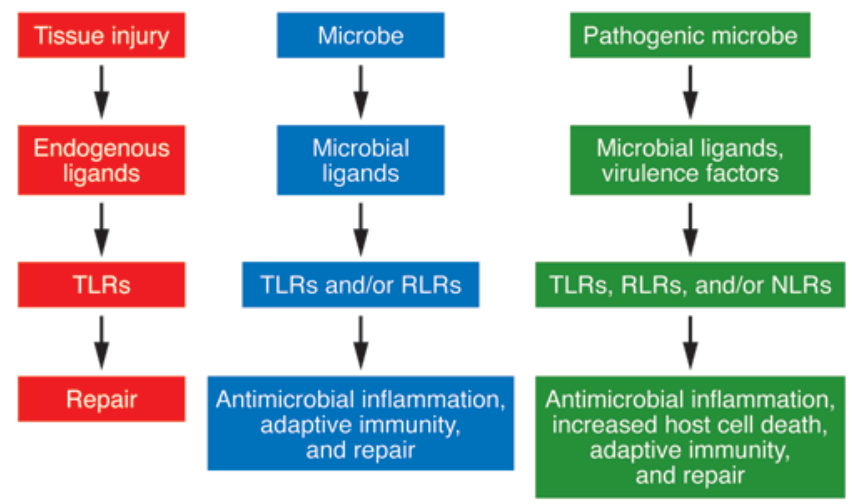

ii

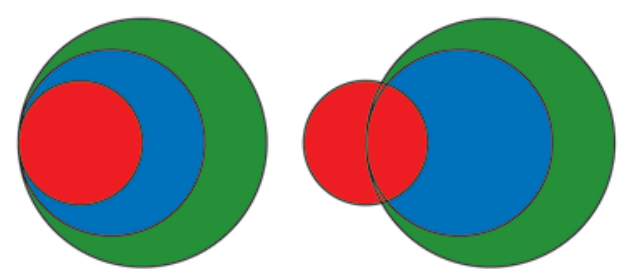

might play a role in amplifying inflammatory signals, but by themselves might lead to a qualitatively different response that favors healing. Mechanistically, endogenous ligands might be weak stimulators that induce a partial signal during tissue damage. Alternatively, there might be additional, TLR-independent signaling pathways induced by tissue damage that direct the TLRdependent response toward repair. This scenario would be conceptually related to the crosstalk between the TLR and NLR pathways. Microbial TLR signaling would need to be dominant over these modulating pathways, however, as premature tissue repair before an infection is resolved must be avoided. Of course, these two models are not mutually exclusive and other possibilities can be envisioned. One consequence of inflammation that might differ between triggers is the induction of adaptive immunity. In the case of sterile injury, there are no foreign antigens, so activation of $\mathrm{T}$ and $\mathrm{B}$ lymphocytes is irrelevant. Interestingly, proresolution signals such as lipoxins can block IL-12 induction (72). Thus in the absence of microbial infection the healing response might inhibit induction of adaptive immunity.

Mechanisms of differential gene induction via innate receptors. To test the hypothesis that specific stimuli induce distinct inflammatory outcomes, the molecular basis of gene regulation during these different scenarios must be dissected. The mechanisms that lead to specific gene induction downstream of TLRs and other innate receptors are not well understood. Most pathways converge on a common set of transcription factors (e.g., NF- $\kappa B$, IRFs, and AP-1), which makes it challenging to envision how specificity can be generated. Recent progress in this area suggests that answers might come from the study of chromatin modifications at the loci of key proinflammatory or tissue repair genes. Several groups have identified distinct classes of TLR4-induced genes in macrophages based on patterns of chromatin regulation $(68,70$, 73). One discriminator involves the dependence on transcriptional regulators such as the SWI/SNF (switching-defective/sucrose non-fermenting) class of nucleosome-remodeling complexes. The loci of primary response genes do not require the activity

\section{Figure 3}

A simple model for specific gene induction in response to different inflammatory triggers. (i) The known triggers leading to inflammation converge on similar innate receptor pathways. Whether these triggers can be distinguished by the innate immune system and lead to qualitatively distinct responses (as shown here, with blue indicating activation of genes encoding proteins that mediate repair; red indicating activation of genes encoding proteins that mediate antimicrobial inflammation, adaptive immunity, and repair; and green indicating activation of genes encoding proteins that mediate antimicrobial inflammation, increased host cell death, adaptive immunity, and repair) is an important unanswered question. (ii) Differential gene induction may lead to escalating responses and additional genes. Left: In this scenario it is possible that the genes induced by tissue damage (blue) and microbial infection (red) are subsets of the full response induced by pathogenic microbes (green). Right: Alternatively, tissue repair (blue) might induce a qualitatively distinct response to microbial infection (red) and pathogenic microbes (green), skewed toward repair. See text for discussion.

of SWI/SNF complexes, whereas the loci of secondary response genes must be modified by these complexes before transcription can occur (70). For example, Tnfa is classified as a primary gene whose induction downstream of TLR4-initiated signaling does not require nucleosome remodeling by SWI/SNF. In contrast, Il12-p40, a secondary response gene, requires SWI/SNF to remodel nucleosome structures within this locus, to enable certain promoter elements to become accessible to transcription factors necessary for gene expression. A second level of classification involves the regulation of gene expression in response to repeated stimulation. Tnfa is a classic example of a "tolerizable" gene; that is, although macrophages produce high levels of TNF- $\alpha$ the first time they are stimulated with LPS, they do not produce TNF- $\alpha$ when stimulated with LPS for a second (and third) time. Although the expression of certain genes is shut down upon repeated stimulation, other genes remain responsive to secondary TLR4 signaling (68). For example, the gene encoding cathelicidin antimicrobial peptide ( $\mathrm{Cnlp}$ ) is not subject to tolerance and continues to be expressed by macrophages repeatedly stimulated with LPS. In fact, Cnlp represents a class of genes that is induced more strongly following a second stimulation with LPS and, if originally classified as a secondary gene, no longer requires SWI/ SNF complexes for expression (68). This work might be highly relevant to the discussion of specificity within inflammation for at least two reasons. First, it provides a mechanism by which genes can be differentially regulated downstream of the same TLR. Second, it suggests this differential regulation might have evolved to control the expression of distinct functional classes of genes. In the example discussed above, proinflammatory genes such as Tnfa are tolerized, whereas antimicrobial peptides continue to be produced, as they help control microbial infection without risking unnecessary inflammatory disease. This type of regulation might account for specific induction of repair genes in the context of sterile injury. For example, induction of proresolution genes such as those encoding lipoxins and SLPI might occur more rapidly in the absence of microbial TLR ligands.

A final level of specificity might come from contextual inputs gathered from the location of PRR signaling. The clearest example of this phenomenon is the activation of NLR pathways by pathogens that access the cytosol. Although the TLR and NLR pathways can be coordinately activated, they each sample distinct subcellular compartments, so activation of these pathways provides 
contextual information regarding the nature of the microbial challenge to the host (Figure 2). Specifically, the activation of the NLR pathway is interpreted as an indication that a pathogen has gained access to the cytosol, a disturbing development for the host that necessitates a qualitatively distinct response. Such access can occur when microbes disrupt the phagosome and replicate in the cytosol or, alternatively, when pathogens use secretion systems to inject effector proteins into the cytosol to manipulate host cells. Both events are quite threatening, as they indicate the presence of a microbe with an agenda; that is, a microbe with virulence factors that can manipulate the host response. Therefore, activation of NLR family members is interpreted as a much more severe situation for the host than TLR activation alone. Accordingly, activation of the inflammasome results not just in IL-1 processing and secretion but also induces a form of rapid cell death more related to necrosis than apoptosis. It has been suggested that the rationale behind this host-driven cell death is that the pathogens are deprived of the safer intracellular environment in which they can hide from host defenses $(25,26)$. For example, macrophages infected with Legionella pneumophila undergo a rapid cell death that is dependent on detection of cytosolic flagellin by the NLR family members Naip5 and ICE-protease activating factor (Ipaf) $(26,74,75)$. Rapid macrophage death restricts L. pneumophila growth. Similar pathways contribute to the restriction of growth for a number of bacterial pathogens, although the NLR family members involved in sensing the pathogenic bacteria vary, as do their molecular targets (11). Although this rapid cell death helps contain certain pathogenic bacteria, it can also promote inflammation by releasing the contents of cells without the ordered, antiinflammatory events characteristic of apoptotic cell death (24). The proinflammatory nature of this response is an interesting byproduct of this innate immune pathway that restricts pathogenic bacterial cell growth. Whether the proinflammatory signals resulting from cell death also help contain infection has not yet been tested experimentally.

\section{Perspectives and challenges}

Information gathered from the activation of innate receptors by distinct ligands and within distinct compartments can be integrated into a decision about the appropriate degree of inflammation (Figure 3). This decision is likely to involve complex control of target genes through differential signaling or through local epigenetic mechanisms, although many of the mechanistic details responsible for this specificity remain undefined. These inputs might lead to qualitatively different inflammatory outcomes based on whether the trigger is sterile tissue damage, microbial infection, or pathogen infection. In light of this model, it is interesting to note that IL-1 might be responsible for many of the damaging aspects of inflammation. The fact that multiple microbial inputs are required for the optimal induction of this cytokine suggests that not all triggers of inflammation must induce the same degree of damage. By dissecting how specific gene induction is achieved by innate immune signaling pathways, we can begin to discover how to manipulate these different degrees of inflammation.

\section{Acknowledgments}

I thank Russell Vance, Dan Portnoy, and members of my lab for discussions.

Address correspondence to: Gregory M. Barton, Division of Immunology, Department of Molecular and Cell Biology, University of California, Berkeley, 401 Life Sciences Addition (MC 3200), Berkeley, California 94720-3200, USA. Phone: (510) 642-2083; Fax: (510) 642-1386; E-mail: barton@berkeley.edu.
1. Kumar, V., Abbas, A.K., Fausto, N., and Mitchell, M. 2007. Robbins basic pathology. W.B. Saunders. Philadelphia, Pennsylvania, USA. 960 pp.

2. Nathan, C. 2002. Points of control in inflammation. Nature. 420:846-852.

3. Segal, B.H., Leto, T.L., Gallin, J.I., Malech, H.L., and Holland, S.M. 2000. Genetic, biochemical, and clinical features of chronic granulomatous disease. Medicine. 79:170-200.

4. Medzhitov, R. 2007. Recognition of microorganisms and activation of the immune response. Nature. 449:819-826.

5. Takeda, K., Kaisho, T., and Akira, S. 2003. Toll-like receptors. Annu. Rev. Immunol. 21:335-376.

6. Bianchi, M.E. 2007. DAMPs, PAMPs and alarmins: all we need to know about danger. J. Leuk. Biol. 81:1-5.

7. Miyake, K. 2007. Innate immune sensing of pathogens and danger signals by cell surface Toll-like receptors. Semin. Immunol. 19:3-10.

8. Matzinger, P. 1994. Tolerance, danger, and the extended family. Annu. Rev. Immunol. 12:991-1045.

9. Nathan, C. 2006. Neutrophils and immunity: challenges and opportunities. Nat. Rev. Immunol. 6:173-182.

10. Cookson, B.T., and Brennan, M.A. 2001. Proinflammatory programmed cell death. Trends Microbiol. 9:113-114.

11. Mariathasan, S., and Monack, D.M. 2007. Inflammasome adaptors and sensors: intracellular regulators of infection and inflammation. Nat. Rev. Immunol. 7:31-40.

12. Goldrath, A.W., and Bevan, M.J. 1999. Selecting and maintaining a diverse T-cell repertoire. Nature. 402:255-262.
13. Medzhitov, R., and Janeway, C.A., Jr. 1997. Innate immunity: the virtues of a nonclonal system of recognition. Cell. 91:295-298.

14. Janeway, C.A., Jr. 1989. Approaching the asymptote? Evolution and revolution in immunology. Cold Spring Harb. Symp. Quant. Biol. 54:1-13.

15. Akira, S., Takeda, K., and Kaisho, T. 2001. Tolllike receptors: critical proteins linking innate and acquired immunity. Nat. Immunol. 2:675-680.

16. Medzhitov, R. 2001. Toll-like receptors and innate immunity. Nat. Rev. Immunol. 1:135-145.

17. Hoffmann, J.A., Kafatos, F.C., Janeway, C.A., Jr., and Ezekowitz, R.A. 1999. Phylogenetic perspectives in innate immunity. Science. 284:1313-1318.

18. Fraser, I.P., Koziel, H., and Ezekowitz, R.A.B. 1998. The serum mannose-binding protein and the macrophage mannose receptor are pattern recognition molecules that link innate and adaptive immunity. Semin. Immunol. 10:363-372.

19. Kawai, T., and Akira, S. 2007. Antiviral signaling through pattern recognition receptors. J. Biochem. 141:137-145

20. Inohara, N., Chamaillard, M., McDonald, C., and Nunez, G. 2005. NOD-LRR proteins: role in hostmicrobial interactions and inflammatory disease. Annu. Rev. Biochem. 74:355-383.

21. Ting, J.P., Kastner, D.L., Hoffman, H.M. 2006. CATERPILLERs, pyrin and hereditary immunological disorders. Nat. Rev. Immunol. 6:183-195.

22. Strober, W., Murray, P.J., Kitani, A., and Watanabe, T. 2006. Signalling pathways and molecular interactions of NOD1 and NOD2. Nat. Rev. Immunol. 6:9-20.

23. Kobayashi, K.S., et al. 2005. Nod2-dependent regulation of innate and adaptive immunity in the intestinal tract. Science. 307:731-734

24. Brennan, M.A., and Cookson, B.T. 2000. Salmonella induces macrophage death by caspase-1-dependent necrosis. Mol. Microbiol. 38:31-40.

25. Derre, I., and Isberg, R.R. 2004. Macrophages from mice with the restrictive Lgn1 allele exhibit multifactorial resistance to Legionella pneumophila. Infect. Immun. 72:6221-6229.

26. Ren, T., Zamboni, D.S., Roy, C.R., Dietrich, W.F., and Vance, R.E. 2006. Flagellin-deficient Legionella mutants evade caspase-1- and Naip5-mediated macrophage immunity. PLoS Pathog. 2:e18.

27. Chen, C.J., et al. 2007. Identification of a key pathway required for the sterile inflammatory response triggered by dying cells. Nat. Med. 13:851-856.

28. Yoneyama, M., et al. 2004. The RNA helicase RIG-I has an essential function in double-stranded RNAinduced innate antiviral responses. Nat. Immunol. 5:730-737.

29. Kawai, T., and Akira, S. 2006. Innate immune recognition of viral infection. Nat. Immunol. 7:131-137.

30. Stetson, D.B., and Medzhitov, R. 2006. Type I interferons in host defense. Immunity. 25:373-381.

31. Ishii, K.J., et al. 2006. A Toll-like receptor-independent antiviral response induced by double-stranded B-form DNA. Nat. Immunol. 7:40-48.

32. Okabe, Y., Kawane, K., Akira, S., Taniguchi, T., and Nagata, S. 2005. Toll-like receptor-independent gene induction program activated by mammalian DNA escaped from apoptotic DNA degradation. J. Exp. Med. 202:1333-1339.

33. Stetson, D.B., and Medzhitov, R. 2006. Recognition of cytosolic DNA activates an IRF3-dependent innate immune response. Immunity. 24:93-103.

34. Takaoka, A., et al. 2007. DAI (DLM-1/ZBP1) is a 
cytosolic DNA sensor and an activator of innate immune response. Nature. 448:501-505.

35. Auerbuch, V., Brockstedt, D.G., Meyer-Morse, N., O'Riordan, M., and Portnoy, D.A. 2004. Mice lacking the type I interferon receptor are resistant to Listeria monocytogenes. J. Exp. Med. 200:527-533.

36. O'Riordan, M., Yi, C.H., Gonzales, R., Lee, K.D., and Portnoy, D.A. 2002. Innate recognition of bacteria by a macrophage cytosolic surveillance pathway. Proc. Natl. Acad. Sci. U. S. A. 99:13861-13866.

37. Mellman, I., and Steinman, R.M. 2001. Dendritic cells: specialized and regulated antigen processing machines. Cell. 106:255-258.

38. Malaviya, R., Ikeda, T., Ross, E., and Abraham, S.N 1996. Mast cell modulation of neutrophil influx and bacterial clearance at sites of infection through TNF-alpha. Nature. 381:77-80.

39. Nathan, C., and Shiloh, M.U. 2000. Reactive oxygen and nitrogen intermediates in the relationship between mammalian hosts and microbial pathogens. Proc. Natl. Acad. Sci. U. S. A. 97:8841-8848.

40. Belaaouaj, A., et al. 1998. Mice lacking neutrophil elastase reveal impaired host defense against gram negative bacterial sepsis. Nat. Med. 4:615-618.

41. Reeves, E.P., et al. 2002. Killing activity of neutrophils is mediated through activation of proteases by K+ flux. Nature. 416:291-297.

42. Tkalcevic, J., et al. 2000. Impaired immunity and enhanced resistance to endotoxin in the absence of neutrophil elastase and cathepsin G. Immunity. 12:201-210.

43. Levy, B.D., Clish, C.B., Schmidt, B., Gronert, K., and Serhan, C.N. 2001. Lipid mediator class switching during acute inflammation: signals in resolution. Nat. Immunol. 2:612-619.

44. Serhan, C.N. 2007. Resolution phase of inflammation: novel endogenous anti-inflammatory and proresolving lipid mediators and pathways. Annu. Rev. Immunol. 25:101-137.

45. Serhan, C.N., and Savill, J. 2005. Resolution of inflammation: the beginning programs the end. Nat. Immunol. 6:1191-1197.

46. Jin, F.-Y., Nathan, C., Radzioch, D., and Ding, A. 1997. Secretory leukocyte protease inhibitor: a macrophage product induced by and antagonistic to bacterial lipopolysaccharide. Cell. 88:417-426.

47. Ashcroft, G.S., et al. 2000. Secretory leukocyte protease inhibitor mediates non-redundant func- tions necessary for normal wound healing. Nat. Med. 6:1147-1153.

48. Giannoudis, P.V. 2003. Current concepts of the inflammatory response after major trauma: an update. Injury. 34:397-404.

49. Jiang, D., et al. 2005. Regulation of lung injury and repair by Toll-like receptors and hyaluronan. Nat. Med. 11:1173-1179.

50. Mollen, K.P., et al. 2006. Emerging paradigm: Tolllike receptor 4 - sentinel for the detection of tissue damage. Shock. 26:430-437.

51. Teder, P., et al. 2002. Resolution of lung inflammation by CD44. Science. 296:155-158.

52. Gallucci, S., Lolkema, M., and Matzinger, P. 1999. Natural adjuvants: endogenous activators of dendritic cells. Nat. Med. 5:1249-1255.

53. Wu, H., et al. 2007. TLR4 activation mediates kidney ischemia/reperfusion injury. J. Clin. Invest. 117:2847-2859.

54. Biragyn, A., et al. 2002. Toll-like receptor 4-dependent activation of dendritic cells by beta-defensin 2 . Science. 298:1025-1029.

55. Guillot, L., et al. 2002. The immunostimulatory activity of the lung surfactant protein-A involves Toll-like receptor 4. J. Immunol. 168:5989-5992.

56. Lande, R., et al. 2007. Plasmacytoid dendritic cells sense self-DNA coupled with antimicrobial peptide. Nature. 449:564-569.

57. Leadbetter, E.A., et al. 2002. Chromatin-IgG complexes activate B cells by dual engagement of IgM and Toll-like receptors. Nature. 416:603-607.

58. Ohashi, K., Burkart, V., Flohe, S., and Kolb, H. 2000. Heat shock protein 60 is a putative endogenous ligand of the Toll-like receptor- 4 complex. J. Immunol. 164:558-561.

59. Park, J.S., et al. 2006. High mobility group box 1 protein interacts with multiple Toll-like receptors. Am. J. Physiol. Cell Physiol. 290: C917-C924.

60. Shi, Y., Evans, J.E., Rock, K.L. 2003. Molecular identification of a danger signal that alerts the immune system to dying cells. Nature. 425:516-521.

61. Tsung, A., et al. 2007. HMGB1 release induced by liver ischemia involves Toll-like receptor 4 dependent reactive oxygen species production and calciummediated signaling. J. Exp. Med. 204:2913-2923.

62. Vabulas, R.M., et al. 2002. HSP70 as endogenous stimulus of the Toll/interleukin-1 receptor signal pathway. J. Biol. Chem. 277:15107-15112.
63. Tsan, M.F., and Baochong, G.. 2007. Pathogenassociated molecular pattern contamination as putative endogenous ligands of Toll-like receptors. J. Endotoxin Res. 13:6-14.

64. Marshak-Rothstein, A. 2006. Toll-like receptors in systemic autoimmune disease. Nat. Rev. Immunol. 6:823-835.

65. Ivanov, S., et al. 2007. A novel role for HMGB1 in TLR9mediated inflammatory responses to CPG-DNA. Blood. 110:1970-1981.

66. Tian, J., et al. 2007. Toll-like receptor 9-dependent activation by DNA-containing immune complexes is mediated by HMGB1 and RAGE. Nat. Immunol. 8:487-496.

67. Wang, H., et al. 1999. HMG-1 as a late mediator of endotoxin lethality in mice. Science. 285:248-251.

68. Foster, S.L., Hargreaves, D.C., and Medzhitov, R. 2007. Gene-specific control of inflammation by TLR-induced chromatin modifications. Nature. 447:972-978.

69. Georgel, P., et al. 2007. Vesicular stomatitis virus glycoprotein $G$ activates a specific antiviral Tolllike receptor 4-dependent pathway. Virology. 362:304-313

70. Ramirez-Carrozzi, V.R., et al. 2006. Selective and antagonistic functions of SWI/SNF and Mi-2beta nucleosome remodeling complexes during an inflammatory response. Genes Dev. 20:282-296.

71. Li, M., et al. 2001. An essential role of the NFkappa B/Toll-like receptor pathway in induction of inflammatory and tissue-repair gene expression by necrotic cells. J. Immunol. 166:7128-7135.

72. Aliberti, J., Serhan, C., and Sher, A. 2002. Parasite-induced lipoxin A4 is an endogenous regulator of IL-12 production and immunopathology in Toxoplasma gondii infection. J. Exp. Med. 196:1253-1262.

73. Weinmann, A.S., et al. 2001. Nucleosome remodeling at the IL-12 400 promoter is a TLR-dependent, Rel-independent event. Nat. Immunol. 2:51-57.

74. Zamboni, D.S., et al. 2006. The Birc1e cytosolic pattern-recognition receptor contributes to the detection and control of Legionella pneumophila infection. Nat. Immunol. 7:318-325.

75. Molofsky, A.B., et al. 2006. Cytosolic recognition of flagellin by mouse macrophages restricts Legionella pneumophila infection. J. Exp. Med. 203:1093-1104. 